\title{
Experiencia de construcción de referentes curriculares con incorporación tecnológica para la formación del profesorado de América Latina y el Caribe
}

\author{
William Oswaldo Flores López
}

\section{Resumen}

F $\mathrm{n}$ este artículo se ha presentado la experiencia de construcción de referentes curriculares con incorEoración tecnológica para la formación del profesorado en y para la diversidad de América Latina y el Caribe. Fue un estudio cualitativo con un enfoque hermenéutico donde se ha expuesto el contexto, la metodología y el trabajo que posibilitaron la producción de un conjunto de referentes curriculares con la participación de profesores investigadores de las universidades de México, El Salvador, Nicaragua, Colombia, Perú, Bolivia, Chile y España. Los resultados muestran que los referentes curriculares se centran en la formación de profesores que puedan contribuir a superar las desigualdades económicas, sociales y culturales que inciden en el contexto escolar y en el aprendizaje. Además de contemplar y resaltar el reconocimiento, la acogida y el trabajo con la diversidad, como expresión comunicativa que suprime distancias y permite la creación de productos abiertos e interactivos para poblaciones vulnerables de América Latina y el Caribe.

Palabras clave: Referentes Curriculares; Formación de Profesores; Tecnología; Diversidad.

\section{Abstract}

This article presents the experience of construction of curriculum referents with technological incorporation for teachers' training in Latin America and the Caribbean in and for the diversity of Latin America and the Caribbean. It was a qualitative study with a hermeneutical approach where it was exposed the context, the methodology and the work that enabled the production of a set of curricular referents with the participation of research professors from the universities of Mexico, El Salvador, Nicaragua, Colombia, Peru, Bolivia, Chile and Spain. The results show that the curricular referents are focus on the teachers' training who can contribute to overcome the economic, social and cultural inequalities that affect the school and learning context. In addition to contemplate and highlight the recognition, acceptance and work with diversity, as communicative expression that suppresses distances and allows the creation of open and interactive products for vulnerable populations in Latin America and the Caribbean.

Keywords: Curriculum referents; Teachers' training; Technology; Diversity.

\footnotetext{
Doctor en Educación. Profesor Investigador de la Universidad de las Regiones Autónomas de la Costa Caribe Nicaragüense.

Correo: william.flores@uraccan.edu.ni
}

Recibido: 05/06/2017 - Aprobado: 23/06/2017 


\section{Introducción}

Esta investigación describe el contexto, la metodología y el trabajo que posibilitaron la producción de un conjunto de referentes curriculares con incorporación tecnológica para la formación del profesorado de Ciencias, Lenguaje y Comunicación, asimismo de la Matemática en y para la diversidad en el marco del proyecto ALTER-NATIVA con la participación de profesores investigadores de universidades de México, El Salvador, Nicaragua, Colombia, Perú, Bolivia, Chile y España.

Cabe mencionar que, el proyecto ALTERNATIVA responde al llamado de las políticas mundiales, regionales y locales a la educación para promover la formación docente, el desarrollo de estrategias y la recreación de ambientes que, en su conjunto ofrezcan condiciones para una educación para todos a lo largo de toda la vida (UNESCO, 1996; CRES, 2008). En este sentido, las acciones principales de ALTER-NATIVA han sido: i) La construcción consensuada y validada de referentes curriculares generales para programas de formación de profesores en las áreas de lenguaje, matemáticas y ciencias y, a partir de ellos, ii) Proponer diseños didácticos diferenciados para contextos de diversidad, incorporando las Tecnologías de la Información y la Comunicación. Tales acciones se llevaron a cabo con la participación activa de las universidades promotoras de los países asociados en ALTER-NATIVA como red interinstitucional y transdisciplinaria, que proporciona un ambiente para los profesores, de reflexión y acción educativa permanente, con capacidad para atender poblaciones en la educación básica y para favorecer el acceso y la permanencia de personas con Necesidades Educativas Diversas que ingresen a los programas ofrecidos por las universidades asociadas.

\section{Contexto educativo de América Latina y el Caribe}

La red ALTER-NATIVA identificó un problema común de exclusión educativa y de vulnerabilidad sociocultural para las poblaciones en contexto de diversidad, como es formar parte de las tasas de analfabetismo en América Latina y el Caribe (ALC); bajo acceso a la educación básica, media y superior; acceso a la escolaridad en condición de extra edad; aumentando la brecha en el desarrollo sociocognitivo del estudiantado con necesidades educativas diversas; y altos índices de deserción de estos estu- diantes (León, García, Calderón, \& Soler, 2013). Así mismo, se identificaron problemas relacionados con la formación de profesores en ALC y con sus estatus socio profesional; es decir, la falta de profesionales en su formación académica, profesional y precarias condiciones económicas, todo lo afecta su desempeño y su autoestima.

Los destinatarios de la propuesta ALTERNATIVA son, en primera instancia, el profesorado y el estudiantado de las facultades de educación en las áreas de Ciencias Naturales, Lenguaje y Comunicación, y Matemática; y de las facultades de ingeniería; informática o sistemas de las universidades involucradas en el proyecto; $y$, por extensión, los ministerios de educación y las secretarías de educación de Iberoamérica, encargadas de las políticas educativas para la diversidad. Los beneficiarios finales fueron las personas con condiciones auditiva y visuales diversas, personas de comunidades indígenas, organizaciones educativas y estudiantado, en general, que verán incrementadas sus opciones de participación en los procesos de enseñanza y aprendizaje.

Con respecto a la población indígena, se sabe que oscila entre 34 y 40 millones o el equivalente a los porcentajes del 8 o el 10 de la población total en América Latina y el Caribe. De este total, Alter-Nativa representa a cinco países: Bolivia, Colombia, México, Nicaragua y Perú, cuyos habitantes indígenas, en conjunto, superan los 20 millones de personas es decir aproximadamente el 50 por ciento de la población indígena en América Latina y el Caribe (Calderón, 2014). Estos pueblos viven una situación crítica, debido a las condiciones de pobreza - revelada en los indicadores de nutrición, salud, la exclusión de género, entre otros - y, por ello, de marginación de la educación - analfabetismo que abarca pueblos cuyas lenguas maternas están ya extinguidas-, exclusión social y marginación política que data desde los tiempos coloniales (León, García, Calderón y Soler, 2013). Esta situación pone de manifiesto el desmedro de sus culturas, el repliegue del uso de sus lenguas maternas frente al español como lengua dominante, lo que normalmente genera un problema para el reconocimiento, la construcción y el mantenimiento de las identidades de estos pueblos, convirtiéndolos en "[...] uno de los segmentos sociales con mayor vulnerabilidad y que plantea un enorme reto para su atención educativa" (Calderón, 2014). 
La población sorda — perteneciente o no a población indígena o de otras culturas—, además de la marginación económica y cultural, manifiesta falta de oportunidades de comunicación y de participación social, política y educativa, lo cual genera que se presenten dos problemáticas: 1) La concepción de la sordera como una de ciencia biológica (Colombia y El Salvador), lo que conduce al estigma social causante del ocultamiento de las personas con esta condición por parte de la propia familia (León, García, Calderón, \& Soler, 2013), y 2) Las propuestas educativas centradas en respuestas clínicas y normalizantes para la educación de la persona sorda (Skliar, 2008).

En relación con la población con condición visual diversa, se destaca la falta de cobertura educativa y la baja calidad de la atención educativa, que incluye a la población adulta iletrada. Adicionalmente, prevalece una problemática de tipo cultural con respecto a la persona con diferencia visual: la estigmatización intelectual y social por causa de su diversidad y la falta de estudios específicos sobre el acceso de la persona con limitaciones visuales (LV) a la vida escolar (Calderón, 2014).

Finalmente, se considera que la acción emprendida por ALTER-NATIVA constituye un acto político. Representa la puesta en escena de la voz del profesorado, de formadores de profesores y de profesores en formación y en ejercicio, hablando desde y para América Latina y el Caribe. Se trata de pensar las diferencias de nuestras propias diferencias y construir con ellas, una opción para nuestra educación (León, García, Calderón, \& Soler, 2013).

\section{Materiales y métodos}

El presente estudio se enmarca en el paradigma cualitativo porque es una actividad sistemática orientada a la comprensión en profundidad de fenómenos educativos y sociales, a la transformación de prácticas y escenarios socioeducativos, a la toma de decisiones y también hacia el descubrimiento y desarrollo de un cuerpo organizado de conocimiento. Desde esta perspectiva, Hernández et al. (2014), explican que en el paradigma cualitativo el enfoque o diseño "es el abordaje general que utilizará el investigador en el proceso de investigación" (p. 492). En este sentido, esta investigación tiene un enfoque hermenéutico, porque se interpretaron los significados de textos y acciones en el proceso educativo.

Por tales razones, se utilizó como método el análisis de contenido de los textos, el cual ayudó a describir los documentos en sus partes esenciales. Para ello, se empleó la técnica de revisión de documentos, donde nos centramos en los argumentos, razones, propósitos y los significados que los mismos presentan sobre los referentes curriculares. Por consiguiente, se construyó una guía de revisión documental como instrumento, que permitió analizar los libros, artículos científicos, artículos de prensa, leyes, políticas, normas institucionales y sitios web.

\section{Resultados y discusión}

\section{Acciones metodológicas en el Proyecto ALTER-NATIVA}

El proyecto ALTER-NATIVA ha tenido por objetivo "formular referentes curriculares ${ }^{2}$ consensuados en la comunidad de impacto del proyecto, para la formación de profesores requeridos para un desarrollo didáctico diferenciado en contextos de diversidad y apoyados en el uso de tecnologías de la información y comunicación como elemento clave en el proceso formativo". En correspondencia a lo anterior, ALTERNATIVA organizó el desarrollo de sus actividades en paquetes de trabajos: gestión del proyecto; red internacional; referentes curriculares; infraestructura tecnológica; guía de integración tecnológica; escenarios de validación; diseño y creación de repositorio de OVA; y difusión y sostenibilidad.

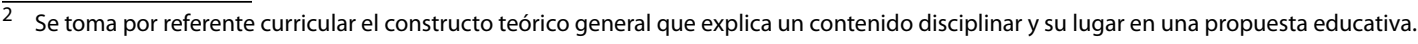


Tabla 1: Paquetes de Trabajos en el Proyecto ALTER-NATIVA

\begin{tabular}{|c|c|c|}
\hline $\begin{array}{l}\text { Nombre } \\
\text { Paquete de } \\
\text { Trabajo (PT) }\end{array}$ & $\begin{array}{l}\text { Socios } \\
\text { responsables }\end{array}$ & Entidades cooperantes \\
\hline PT1: Gestión del proyecto & Universidad Distrital Francisco de José de Caldas de Colombia y Universidad de Girona de España. & \\
\hline $\begin{array}{l}\text { PT2: Red Internacional de Institucio- } \\
\text { nes ALTER-NATIVA }\end{array}$ & Universidades: Distrital FJC de Colombia y Girona de España. & INCI-INSOR Fundación Sidar \\
\hline PT 3. Referentes curriculares & $\begin{array}{l}\text { UDELAS de Panamá, Universidad Distrital Francisco José de Caldas de Colombia, Universidad de } \\
\text { las Regiones Autónomas de la Costa Caribe Nicaragüense de Nicaragua, Universidad de San Mar- } \\
\text { cos de Perú, Universidad Pedagógica Nacional de México, Universidad de San Andrés de Bolivia. }\end{array}$ & INCI-INSOR Colombia \\
\hline PT4: Infraestructura tecnológica & $\begin{array}{l}\text { Universidad Nova de Portugal, Universidad Distrital Francisco José de Caldas de Colombia y Uni- } \\
\text { versidad de Girona de España. }\end{array}$ & \\
\hline PT5: Guías de integración en TIC & $\begin{array}{l}\text { Universidades: UNED de España, Pontifica Universidad Católica de Valparaíso de Chile, Universi- } \\
\text { dad de las Regiones Autónomas de la Costa Caribe Nicaragüense de Nicaragua. }\end{array}$ & INCl-Colombia \\
\hline $\begin{array}{l}\text { PT6: Escenarios de validación de re- } \\
\text { ferentes }\end{array}$ & $\begin{array}{l}\text { Universidad Pedagógica Nacional de México, Universidad San Marcos de Perú, Universidad Ma- } \\
\text { yor de San Andrés de Bolivia, Universidad Distrital Francisco José de Caldas de Colombia, UDELAS } \\
\text { de Panamá y Pontifica Universidad Católica de Valparaíso de Chile. Universidad de las Regiones } \\
\text { Autónomas de la Costa Caribe Nicaragüense de Nicaragua. }\end{array}$ & INCI, INSOR- Colombia \\
\hline $\begin{array}{l}\text { PT7: Diseño y Creación de un Repo- } \\
\text { sitorio de Objetos de Aprendizaje } \\
\text { Accesible }\end{array}$ & Universidades: San Juan de Argentina y UNED. & Fundación Sidar \\
\hline PT8: Difusión y sostenibilidad & $\begin{array}{l}\text { Universidades: Pontifica Universidad Católica de Valparaíso de Chile, Pedagógica Nacional de } \\
\text { México, San Marcos de Perú, Mayor de San Andrés de Bolivia y Distrital FJC de Colombia. }\end{array}$ & \\
\hline
\end{tabular}

A partir de la constitución de los paquetes de trabajo (PT), el proyecto ALTER-NATIVA asumió la estrategia de Comunidades de Práctica (Wenger, 2001). En términos teóricos, la comunidad de práctica se constituye si entre sus miembros existe un compromiso mutuo de realizar una cierta empresa, lo que lleva a una práctica compartida en la que se genera aprendizaje de carácter colectivo. En este sentido, ALTER-NATIVA utiliza herramientas colaborativas e interactivas (Plataforma colaborativa; herramientas de google; conferencias virtuales; TIC para la investigación; talleres de formación; y TIC para el seguimiento y evaluación del proyecto) para construir los entregables del proyecto, véase la figura 1.

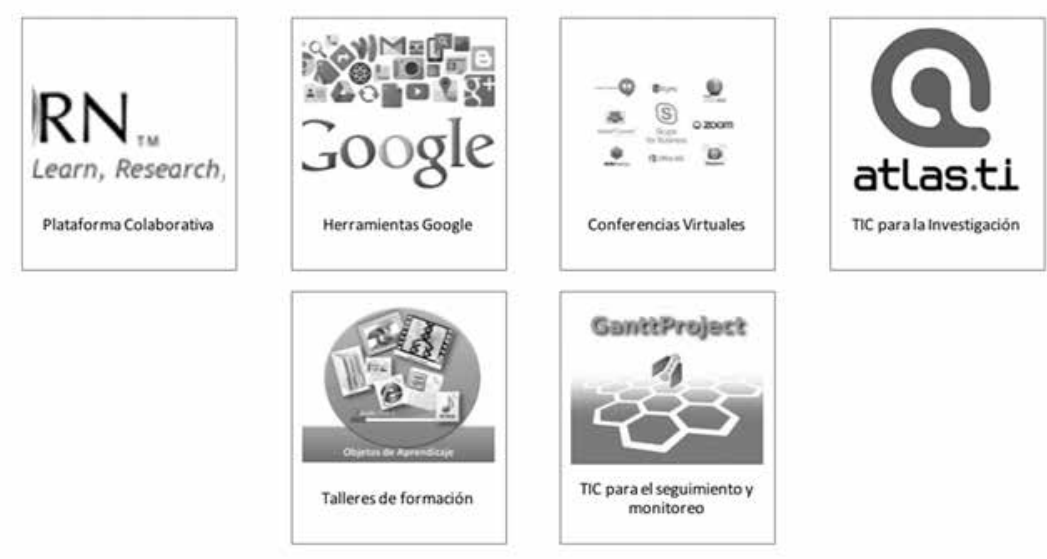

Figura 1: Herramientas colaborativas e interactivas en el proyecto ALTER-NATIVA 
La acción fundamental que realizaron las universidades proponentes de los países asociados para el proyecto ALTER-NATIVA es: construir referentes curriculares generales, de manera consensuada, para las áreas de lenguaje, matemáticas y ciencias para programas de formación de profesores con un enfoque de una educación en y para la diversidad; y a partir de ellos, generar estrategias para el desarrollo de tecnologías aplicadas a exigencias educativas especiales identificadas en poblaciones diversas. El proyecto articuló acciones concretas e inmediatas por parte de las comunidades educativas nacionales e internacionales, que comprometen, fundamentalmente, cinco instancias poco desarrolladas y poco articuladas anteriormente en las propuestas educativas de los países de América Latina y el Caribe, y que se reflejan en la tabla 2.

Tabla 2: Acciones concretas en el proyecto ALTER-NATIVA

\section{ACCIONES CONCRETAS EN EL PROYECTO ALTERNATIVA}

El estudio de invariantes y de variantes curriculares en las áreas de lenguaje, matemáticas y ciencias en programas de formación de profesores, considerando una EDUCACIÓN EN Y PARA LA DIVERSIDAD. Este es un factor de impacto curricular para las instituciones formadoras de profesores.

El diseño tecnológico de ambientes de aprendizaje (en las áreas de lenguaje, matemáticas y ciencias), adecuados a las condiciones y las necesidades de LA DIVERSIDAD de: poblaciones, formas de acceso a los conocimientos y formas de comunicación e interacción en la educación. Para ello se propone el desarrollo de las TIC, como una alternativa para la optimización de los procesos de interacción entre las personas y entre ellas y la información. Se destaca la necesidad de desarrollar aplicaciones tecnológicas específicas al servicio de las propuestas didácticas y de la generación de ambientes de aprendizaje para poblaciones con exigencias educativas particulares (sordera, ceguera, vulnerabilidad social, etc.). De igual modo, se requiere que este tipo de aplicaciones exhiban un alto grado de convencionalidad y de capacidad de uso para distintas comunidades y contextos culturales.

El desarrollo del lenguaje y de los lenguajes como puerta de entrada a todo el aprendizaje escolar y como un modo de participación socio-cultural y de desarrollo de la identidad. Resulta imprescindible considerar factores y modos de comunicación para todos los grupos socio-educativos e integrar, principalmente a los alumnos con necesidades educativas en la diversidad (NEED), desde sus propias diversidades lingüísticas, étnicas, socio-culturales, sensoriales, etc., de tal manera que la brecha y la tasa de analfabetismo (lecto-escritural, académico y digital) en América Latina se reduzcan considerablemente, en pro de sociedades más democráticas y dialogantes.

El desarrollo de los aprendizajes matemáticos y científicos como una forma de comprender y de estar en el mundo. La experiencia socio-cultural en las matemáticas y en las ciencias pone en evidencia la necesidad, tanto para estudiantes como para profesores, del acceso al conocimiento que requieren las prácticas académicas y profesionales. Se hace necesario, en general, que la deserción escolar en la educación básica, media y universitaria deje de estar motivada por las áreas de matemáticas y de ciencias, como se reporta en los informes de evaluación escolar nacionales e internacionales en América Latina (pruebas PISA, entre otras).

Aportar a los programas de formación de formadores, para ampliar el reducido número de profesores con formación para actualizar sus conocimientos profesionales especializados, para comunicarse y desarrollar habilidades en campos específicos del conocimiento, en ambientes educativos que involucran poblaciones con exigencias comunicativas y cognitivas particulares. Al respecto, se requiere dar el salto de una formación de educadores para poblaciones especiales (por discapacidades) hacia una formación de profesores en y para la diversidad, desde los diversos campos del saber.

\section{Referentes curriculares y orientaciones tecnológicas}

Los referentes curriculares y las orientaciones tecnológicas se caracterizan por ser productos realizados con todos y para todos en un diseño didáctico que se estructura a partir de la idea de coexistencia con la diversidad, en una realidad en la que la multidisciplinariedad, la pluritecnología y las diversas condiciones personales y sociales que están a la orden del día (León et al., 2013).
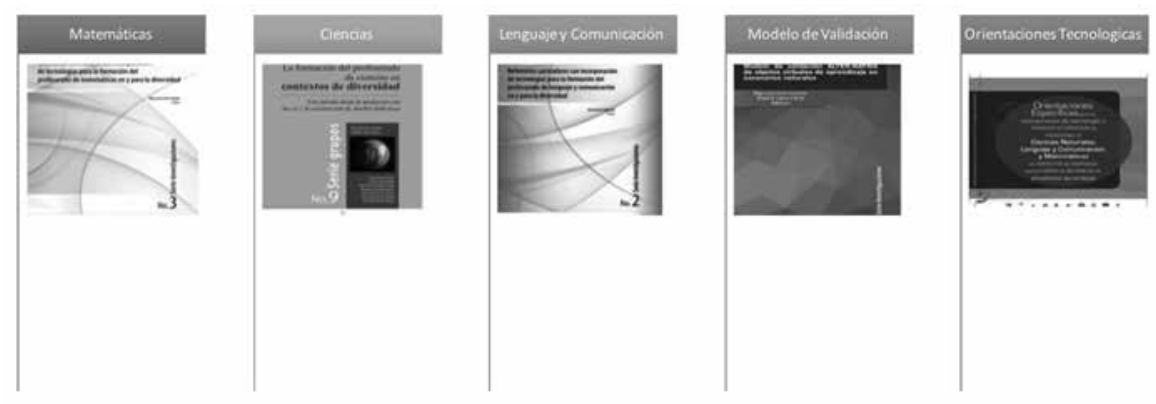

Figura 2: Referentes curriculares con incorporación tecnológica

\footnotetext{
3 Consideramos que las Necesidades Educativas en la Diversidad" son el resultado de una condición física, fisiológica, sensorial o de una de-privación social o cultural que ponen al individuo en una situación de necesidad para el acceso al conocimiento y a las relaciones físico-sociales y culturales.
} 
Referentes curriculares con incorporación tecnológica para la formación del profesorado de Matemáticas en y para la diversidad son el resultado del reconocimiento de cinco aspectos fundamentales: (i) Las múltiples experiencias con lo matemático y su didáctica; (ii) Las múltiples representaciones de lo matemático y de su didáctica; (iii) Los múltiples tipos de interacción en los ambientes de aprendizaje; (iv) Las poblaciones en vulnerabilidad educativa por sus condiciones sensoriales, étnicas o económicas; y (v) Una metodología de interacción y desarrollo definida por las comunidades de práctica (León, 2014).

Referentes curriculares con incorporación tecnológica para la formación de profesorado de Ciencias en y para la diversidad contienen algunas orientaciones para la formación del profesorado de ciencias naturales que atienden poblaciones en contexto diversidad. Es importante mencionar que los referentes curriculares son una posibilidad distinta de asumir la enseñanza de las ciencias naturales, lo que a su vez implica reflexión y evaluación constante de la práctica de los docentes de esta área (García et al., 2014); que los compromete también con los desarrollos tecnológicos actuales, por consiguiente, los referentes curriculares se sitúan como un instrumento para analizar y canalizar esfuerzos para diseñar y gestionar prácticas didácticas con incorporación de las tecnologías lo que conlleva a la construcción de mundos posibles, cercanos a los ideales de participación, igualdad y equidad (Merino, Contreras, \& Borja, 2013).

Referentes curriculares con incorporación tecnológica para la formación del profesorado de Lenguaje y Comunicación en y para la diversidad se caracterizan por reflexionar sobre el lenguaje y comunicación, sobre el aprendizaje y sobre las condiciones de diversidad lingüística, cultural, social, sensorial de todos los actores educativos. En este sentido, los referentes curriculares están orientados específicamente a la enseñanza del español, en contexto de diversidad y con el uso de tecnologías (Calderón, 2014). Además, los referentes curriculares con incorporación tecnológica para la formación del profesorado de lenguaje y comunicación recomiendan la reflexión e inclusión a la hora de formular currículos, diseños didácticos y acciones educativas para futuros profesores del área de lenguaje.

Modelo de validación ALTER-NATIVA de objetos virtuales de aprendizajes en escenarios natura- les se configura a partir de unidades didácticas inmersas en un ambiente virtual de aprendizaje y está conformado por objetos virtuales de aprendizaje; actividades que responden a las particularidades de las poblaciones diversas; Además, que el modelo de validación tienen la intención de llevar a reflexionar y analizar las posibilidades de aprendizaje por parte de todos los estudiantes, incluidos quienes por sus particularidades tienen una condición de discapacidad sensorial, vulnerabilidad económica, social o cultural.

Las orientaciones específicas para la incorporación de tecnologías en procesos de formación de profesores de ciencias naturales, lenguaje y comunicación, y matemáticas en contexto de diversidad para el diseño de secuencia de enseñanza de aprendizaje, consideran que: (1) La diversidad en las poblaciones presupone que el aprendizaje de la práctica de enseñar se da en un marco de actividades que automáticamente acogen la diversidad y las TIC; y (2) El aprendizaje de las prácticas de diseño, gestión y evaluación de ambientes didácticos con incorporación de TIC para el aprendizaje se da en escenarios donde la diversidad de las poblaciones tiene plena manifestación y las condiciones de acceso a la tecnología son motivo de gestión y revisión institucional (León, y otros, 2013).

Entonces estas orientaciones tecnológicas asumen que la formación del profesorado recae en una práctica que enfatiza: (i) La diversidad y la tecnológica en la constitución de ambientes pedagógicos y didácticos; (ii) La didáctica como eje que propone campos de formación específicos en la constitución del sujeto profesor; (iii) La formación del profesorado como el gran eje curricular articulado de la educación como profesión; y (iv) El reconocimiento y la incorporación de las TIC como elemento estratégico para la formación del profesorado.

\section{Ambientes Interculturales y Pluritecnológicos}

Los ambientes interculturales y pluritecnológicos constituyen un sistema educativo y tecnológico que posibilita el diseño para todos y el diseño con todos, es decir, escenarios que pueden hacer posible que las tecnologías y la educación desarrollen una revolución digital en América Latina y el Caribe. Entre ellos, se destacan: (i) El escenario que proporcionan las redes digitales; (ii) El escenario de las redes de inmigrantes; (iii) El escenario de la escuela pública; (iv) El escenario de los derechos humanos; 
y (v) El escenario de la formación del profesorado de ciencias, lenguaje y comunicación, y matemáticas (León et al., 2013).

Por consiguiente, los resultados tecnológicos en el proyecto ALTER-NATIVA consideran como punto de partida la diversidad, por ello, se configura una plataforma accesible para las poblaciones en contexto de diversidad como es ATutor; una guía de configuración de sistemas operativos y navegadores; una serie de objetos virtuales de aprendizajes; una plataforma colaboración; una ontología o tesauro; y un kit tecnológico.
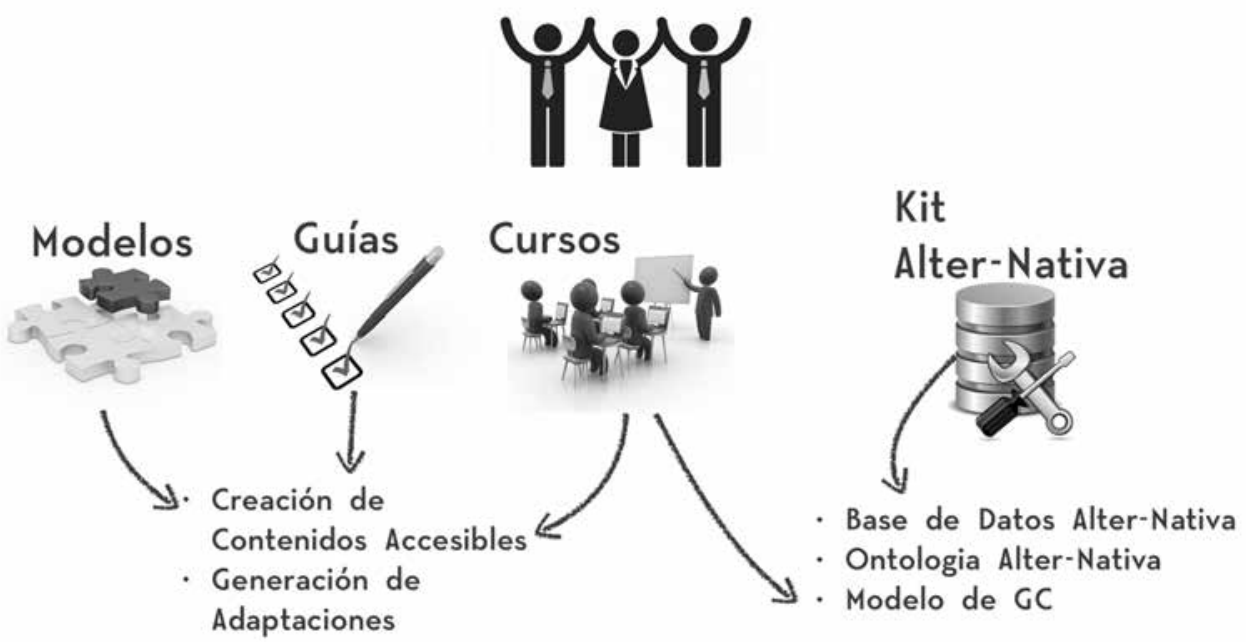

Figura3: Modelos, guías, cursos y Kit ALTER-NATIVA

ATutor: un sistema de gestión de contenidos de aprendizajes de código abierto basado en la web y diseñado con el objetivo de lograr la accesibilidad y adaptabilidad. Como una ayuda a los miembros de las comunidades de práctica y, posteriormente, a los usuarios de ATutor, se diseñó un breve curso de manejo de ATutor cuyo fin último es lograr la familiarización de los usuarios (profesores y estudiantes para profesor) con este ambiente tecnológico. Guía de configuración de sistemas operativos y navegadores: esta guía da inicio con elementos fundamentales como una aproximación al concepto de tecnologías informáticas accesibles, presenta posibles productos de apoyo y finalmente pasa a la presentación de sistemas operativos y navegadores y sus respectivas gamas de configuración para la atención a la diversidad.

Objetos Virtuales de Aprendizajes (OVA): son el último producto de la comunidad del proyecto ALTER-NATIVA y han surgido desde el aprendizaje propio que la comunidad de práctica, es decir, es toda unidad instrumental (documento electrónico, archivo, etc.) y una unidad simbólica portadora de un lenguaje específico y mediadora del aprendizaje (Merino, Contreras, \& Borja, 2013) en concreto, el OVA es una construcción de un diseño con todos y es el punto de llegada de ALTER-NATIVA que partió de la idea de incluir la diversidad y diseñar para ella hasta arribar en el diseño con la diversidad y desde ella. En este sentido, se recomienda las medidas siguientes: (i) Fomentar el conocimiento y el uso de los recursos educativos abiertos; (ii) Crear entornos propicios para el uso de las TIC; (iii) Reforzar la formulación de estrategias y políticas sobre recursos educativos abiertos; (iv) promover conocimientos y la utilización de licencias abiertas; (v) Apoyar el aumento de capacidades para el desarrollo sostenible de materiales de Aprendizaje de calidad; (vi) Impulsar alianzas estratégicas a favor de los recursos educativos abiertos; (vii) promover la elaboración y adaptación de los recursos educativos abiertos en una variedad de idiomas y de contextos culturales; (viii) Alentar la investigación sobre los recursos educativos abiertos; y (ix) Facilitar la búsqueda, la recuperación y el intercambio de recursos educativos abiertos (Flores \& Auzmendi, 2015). 
Plataforma Colabora: es una plataforma de colaboración de la red ALTER-NATIVA. Se sustenta como un repositorio o un compartir de objetos virtuales de aprendizajes y materiales didácticos-tecnológicos para que puedan ser aprovechados en los procesos de formación que implementa el profesorado de educación básica, media, técnica y superior.

Ontología: basada en un tesauro, es decir, en una lista de palabras o términos controlados empleados para representar conceptos. En concreto, el tesauro es un listado que presenta los términos que se utilizan para la representación de ciertos contenidos. Su finalidad es contribuir a la normalización de los términos, de este modo, se facilita la comprensión, la accesibilidad y la comunicación de las poblaciones en contexto de diversidad. Kit Tecnológico: equipos o herramientas tecnológicas basadas en el principio de la accesibilidad y que se utilizan para la operatividad de los referentes curriculares y los ambientes tecnológicos.

\section{Red Institucional de Educación Superior}

Una red internacional de instituciones de Educación Superior de reconocida experiencia en las áreas de pedagogía y desarrollo de tecnología de la información y comunicación con el propósito de desarrollar, mantener y garantizar la continuidad de los productos y servicios generados en el proyecto ALTER-NATIVA.

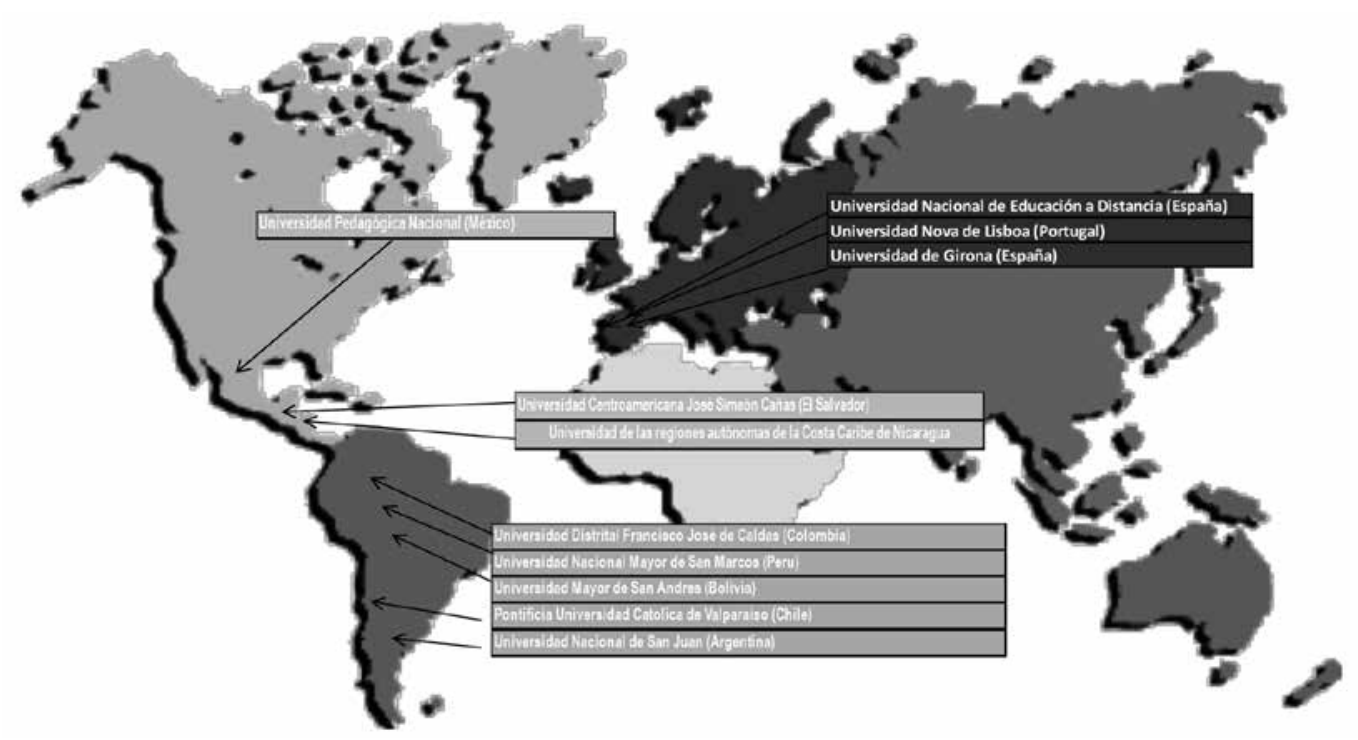

Figura 4: Red ALTER-NATIVA en sus dimensiones educativas y tecnológicas

La misión de la red ALTER-NATIVA es establecer vínculos académicos en red entre instituciones de Educación Superior, entidades cooperantes y personas vinculadas a la educación, formal o informal de poblaciones en contexto de diversidad, desarrollando estrategias que promueven la aplicación de tecnologías de información y la complementariedad entre las áreas pedagógicas y tecnológicas, para mejorar el acceso a oportunidades educativas de calidad y reducir la disparidad en los logros de aprendizaje, la brecha digital, la desigualdad de acceso a oportunidades educativas de calidad; aumentando el nivel de participación de personas en riesgo de exclusión en los procesos educativos.

La red ALTER-NATIVA desarrolla sus actividades a través de proyectos de investigación y cooperación que presentan un ordenamiento disciplinar más acotado a procesos constitutivos donde el eje central es el desarrollar una cultura informada en la educación sobre tecnologías, investigación en el uso de los contenidos. Las acciones de la red, se regirán por los siguientes principios: 
- La diversidad como un derecho humano tanto individual como de grupos. Todas las personas y los grupos sociales tienen derecho al libre desarrollo y a la expresión de su individualidad, como parte de su condición humana y social.

- La red asume la educación como una acción que aplica criterios de pertinencia cultural e inspira políticas de inclusión, de calidad, de equidad, de actualización de los saberes, de ambientes educativos diversificados y de competencias para la convivencia inclusiva.

- La educación ha de reducir la brecha digital, la desigualdad y la exclusión educativa entre poblaciones de distintas características, mediante el uso y la aplicación de las TIC en las prácticas educativas.

- En la red ALTER-NATIVA se configura y proyecta la acción formadora de profesores aptos para atender poblaciones en contexto de diversidad y una forma de ser y de estar en la sociedad; es decir, "ser con"; "actuar con"; y "reconocer a".

Para tal fin, el objetivo central de la red ALTER-NATIVA es "consolidar un espacio colaborativo, en las dimensiones educativas y tecnológicas, como escenario para la conformación y el desarrollo de comunidades de práctica para la integración de tecnologías de la información y comunicación, con el fin de cubrir las necesidades educativas en contextos de diversidad"; este objetivo se concreta a partir de líneas de investigación, es decir, enfoque intra e interdisciplinarios que permiten englobar procesos, prácticas y perspectivas de análisis y definición disciplinaria con énfasis en los aportes de experimentalidad y creatividad expansiva e inclusiva que significa poner la tecnología al servicio de la enseñanza y el aprendizaje con el fin de atender las necesidades y preferencias de las poblaciones en contexto de diversidad. En este sentido, la red ALTER-NATIVA se propone los ámbitos de acción siguiente:

Tabla 4: Ámbitos de acción de la red ALTER-NATIVA

\begin{tabular}{|l|}
\hline \multicolumn{1}{|c|}{ ÁMBITOS DE ACCIÓN } \\
\hline $\begin{array}{l}\text { La cooperación en la constitución de un escenario iberoamericano, adecuado tecnológicamente para la conformación y el desarrollo de comunidades alterna- } \\
\text { tivas de práctica, en el contexto de las necesidades educativas diversas. }\end{array}$ \\
\hline $\begin{array}{l}\text { El desarrollo de vínculos interinstitucionales orientados a la creación, fortalecimiento y sostenibilidad de espacios educativos, formales e informales, que } \\
\text { promuevan la cooperación entre áreas pedagógicas y tecnológicas de dichas instituciones, para la inclusión y permanencia de poblaciones vulnerables; propi- } \\
\text { ciando el reconocimiento de la diversidad. }\end{array}$ \\
\hline $\begin{array}{l}\text { El fomento de la formación de educadores que en su práctica pedagógica privilegien la configuración de una forma de ser y estar en la sociedad, aplicando el } \\
\text { principio de 'ser con','actuar con', y'reconocer a'los grupos con necesidades educativas diversas. }\end{array}$ \\
\hline $\begin{array}{l}\text { El diseño e implementación de estrategias colaborativas de intercambio de información y de resultados de investigaciones pedagógicas, científicas y tecnoló- } \\
\text { gicas, relativas a la misión y objetivos de la Red. }\end{array}$ \\
\hline $\begin{array}{l}\text { El diseño y desarrollo de proyectos educativos orientados a responder a problemáticas de poblaciones con necesidades educativas diversas mediante acciones } \\
\text { pedagógicas, tecnológicas, de difusión, de gestión y de sostenibilidad. }\end{array}$ \\
\hline La aplicación de los referentes curriculares desarrollados en el seno de la Red ALTERNATIVA, en programas de formación de profesores. \\
\hline
\end{tabular}

El fomento del desarrollo de las siguientes acciones complementarias: a) Establecimiento de mecanismos para la articulación de programas y propuestas de formación post-gradual, en los niveles de especialización, maestría y doctorado, en diversas líneas, a partir de las experiencias curriculares de cada una de las universidades de la red, en áreas administrativas, educativas, tecnológicas y científicas; b) Generación de espacios y estrategias de intercambios académicos entre las universidades miembros de la red, como pasantías o estancias cortas; y c) Establecimiento de vínculos con las redes de bibliotecas virtuales existentes entre las universidades miembros de la red ALTER-NATIVA.

\section{Conclusiones}

El objetivo de esta comunicación, consistió en exponer el contexto, la metodología y el trabajo que posibilitaron la producción de un conjunto de referentes curriculares con incorporación tecnológica para la formación del profesorado de ciencias, lenguaje y comunicación, y matemáticas en y para la diversidad. Referentes curriculares que han constituido un proceso de construcción colectiva, es decir, un proceso de participación democrática e inclusiva, donde convergen diferentes instancias y representaciones con el fin 
de planificar, ejecutar y evaluar concertadamente, acciones de formación del profesorado de América Latina y el Caribe.

Es por ello que la metodología, la comunidad de práctica, generó una articulación entre los sistemas educativos de América Latina y el Caribe, contribuyendo a la construcción de los referentes curriculares con incorporación tecnológica para la formación del profesorado de ciencias, lenguaje y comunicación, y matemáticas. Tomando en cuenta la diversidad de las poblaciones de ALC, es decir, el reconocimiento de las relaciones y condiciones del profesorado, estudiantado, de los contextos, de las políticas, de los elementos constitutivos de éstas que hacen de la educación un terreno fértil para el desarrollo humano basado en el respeto, la igualdad y equidad en lo individual y lo colectivo.

El trabajo en comunidad de práctica permitió diseñar, gestionar y evaluar ambientes interculturales y pluritecnológicos, que en el proyecto ALTERNATIVA son una estrategia de autodesarrollo para contrarrestar las condiciones de pobreza y marginación de los pueblos en contexto de diversidad. Brindando oportunidades de accesibilidad al estudiantado y de actualización didáctica-pedagógica y tecnológica al profesorado, así como, condiciones de una educación para todos y con todos a lo largo de toda la vida. A su vez, los ambientes responden al enfoque intercultural, porque contribuyen el desarrollo de un diálogo intercultural como una estrategia para promover espacio de innovación de nuevos conocimientos, ya que confronta elementos de diferentes horizontes y perspectivas culturales, abriendo la posibilidad de impulsar un proceso de complementación y enriquecimiento entre la ciencia moderna y otros saberes (URACCAN, 2013).

También, se constituye la red ALTER-NATIVA, establecida como una acción de complementariedad, es decir, es la comprensión y actuación de forma conjunta y colaborativa que conlleva a la unidad y equilibrio entre la red profesores investigadores y su entorno para potenciar capacidades y alcanzar la formación del profesorado en y para la diversidad. En concreto, se ha constituido una red en sus dimensiones educativas y tecnológicas como un escenario adecuado tecnológicamente para la conformación y el desarrollo de comunidades de práctica educativa en y para la diversidad. La red ALTER-NATIVA se organiza por instituciones de Educación Superior de Europa, América Latina y el Caribe donde participan profesores investigadores especializados en educación, didáctica-pedagogía y tecnología.

Finalmente, el proyecto ALTER-NATIVA configura las bases cognitivas para el desarrollo del proyecto ACACIA 4 que tiene por objetivo "fomentar la integración universitaria a partir del diseño y validación de un sistema de centros de apoyo y desarrollo educativo profesional en América Latina y el Caribe, mediante la cooperación intra e interinstitucional en la producción y desarrollo de recursos didácticos y tecnológicos, y en la generación de estrategias de fomento socioafectivo a poblaciones en riesgo de exclusión universitaria"; En este sentido, ACACIA propone desarrollar Centros de Apoyo y Desarrollo Profesional (CADEP) constituidos en un sistema de módulos: Empodera, Innova, Cultiva, Apoya y Convoca, que además integran laboratorios, software, modelos de sistemas, metodologías, cursos, pautas y orientaciones necesarias para el correcto funcionamiento y desenvolvimiento de cada uno de ellos (ACACIA, 2015).

Además, el CADEP supone un nuevo punto de encuentro y centro de formación y actualización de conocimientos sobre uso y reutilización de tecnologías, creación de contenidos accesibles y generación de las adaptaciones necesarias, didácticas que atienden a las necesidades físicas y sensoriales así como a la afectividad del estudiantado, detección humana y automática de emociones que pueden estar afectando al estudiantado en su avance académico y personal, así como sistemas de gestión del conocimiento para facilitar la interacción interna de los módulos del CADEP y de su relación con otras instancias universitarias internas y externas (Flores et al., 2016).

Agradecimiento: a la Unión Europea, a la RED-ALTER-NATIVA por la información brindada $\mathrm{y}$ al grupo de investigadores de las comunidades de matemáticas, ciencias, y lenguajes y comunicación del proyecto Referentes Curriculares con incorporación tecnológica para facultades de educación en las áreas de Lenguaje, Matemáticas y Ciencias para atender poblaciones en contexto de diversidad.

\footnotetext{
4 Centros de Cooperación para el Fomento, Fortalecimiento y Transferencia de Buenas Prácticas que Apoyan, Cultivan, Adaptan, Comunican, Innovan y Acogen a la Comunidad Universitaria
} 


\section{Bibliografía}

ACACIA. (2015). Centros de Cooperación para el Fomento, Fortalecimiento y Transferencia de Buenas Prácticas que Apoyan, Cultivan, Adaptan, Comunican, Innovan y Acogen a la Comunidad Universitaria. Bogotá: Universidad Distrital Franscico José de Caldas.

Calderón, D. (2014). Referentes curriculares con incorporación técnologica para la formación del profesorado de lenguaje y comunicación. Bogotá: Universidad Distrital Francisco José de Caldas.

CRES. (2008). Declaración de la conferencia regional de la Educación Superior en américa latina y el caribe. Paris: UNESCO.

Flores, W., O., \& Auzmendi, E. (2015). Ambientes Interculturales y Pluritecnológicos Desarrollan el Aprendizaje de las Matemáticas en y para la Diversidad. En C. Varela, A. Miñán, \& L. Bengochea (Ed.), Congreso internacional sobre formación virtual inclusiva y de calidad para el siglo XXI (318-326). Granada: Universidad de Granada.

Flores, W., O., Gutiérrez y Restrepo, E., León, O., Sarraipa, J., Lima, C., Merino, C., Calderón, I., Guinocchio, M., Rivera, M., Calderón, M., \& Boticario, J. (2016). Centros de apoyo y desarrollo educativo profesional para la dismición de la deserción universitaria. Ciencia e Interculturalidad, 18(1), 48-62. DOI: http:// dx.doi.org/10.5377/rci.v18i1.3049

García, Á., Merino, C., Rodríguez, D., Hernández, R., Reyes, F., Abella, L., \& Guevara, J. C. (2014). La formación del profesorado de ciencias en contextos de diversidad: Una mirada desde la mediación con las TIC y la construcción de diseños didácticos. Bogotá: Universidad Distrital Francisco José de Caldas.

Hernández, R., Fernández, C., \& Baptista, M. (2010). Metodología de la Investigación. México: McGra-Hill.
León, O. (2014). Referentes curriculares con incorporación tecnológica para la formación del profesorado de matemáticas en y para la diversidad. Bogotá: Universidad Distrital Francisco José de Caldas.

León, O., García, A., Calderón, D., \& Soler, S. (2013). Referentes curriculares para la formación de profesores en las áreas de ciencias naturales, lenguaje y comunicación y matemáticas para poblaciones en contexto de diversidad. Bogotá: Universidad Francisco José de Caldas.

León, O., Medina, R., Saiz, M., Bonilla, M., Romero, J., Gil, D., Correal, M., Flores, W., O., Rojas, N., Peralta, M., Cavanzo, A., Baca, J., Avila, C., \& Márquez, A. (2013). Relaciones entre "Diseño para Todos" y "Diseño con Todos" en Formación de Profesores de Matemáticas. En A. Moreira, L. Bengochea, \& J. Hilera (Ed.), Congreso Internacional Para una Formación Virtual Accesible y de Calidad (162-169). Portugal: Universidad de Lisboa.

Merino, C., Contreras, D., \& Borja, M. (2013). Orientaciones específicas para la incorporación de tecnología en procesos de formación de profesores de ciencias naturales, lenguaje y comunicación, y matemática en contexto de diversidad para el diseño de secuencias didácticas de enseñanza y aprendizaje. Valparaíso: Pontífica Universidad Católica de Valparaíso.

Skliar, C. (2008). ¿Incluir las diferencias? Sobre un problema mal planteado y una realidad insorpotable. http://www.scielo.org.ar/pdf/ orisoc/v8/v8ao2.pdf.

UNESCO. (1996). La educación encierra un tesoro. Paris: UNESCO.

URACCAN. (2013). Proyecto Educativo Institucional . Managua: URACCAN.

Wenger, E. (2001). Comunidades de Práctica. Aprendizaje, significado e identidad. Buenos Aires: Paidós. 